\title{
Classification of Biomedical Images using Counter Propagation Neural Network
}

\author{
Sangita A. Dubey \\ Research Scholar, \\ Faculty of Engineering, Pacific Academy of \\ Higher Education and Research University, \\ Udaipur
}

\author{
Vijay R. Rathod \\ Head, Dept of Electronics \& Telecom \\ St Xaviers Technical Institute Mumbai
}

\begin{abstract}
In this paper, method for the segmentation, feature extraction \& classification for ultrasonic biomedical images are proposed The method has been applied \& implemented Some sets of descriptors corresponding to texture measurements and geometrical features is extracted for each segmented object and applied to a classifier. The paper reports comparative results of classification of ultrasound images; research experimentation has been implemented on various ultrasound images from the standard datasets. The present experimental study is aimed at comparing the evaluation of image processing algorithms and classification techniques applied on the ultrasound images. Different image segmentation techniques have been proposed here for feature extraction. Currently, there is a large amount of research work going on in the field of automated system for inspection, analysis of ultrasound enhancement, feature extraction and classification. Comparison of other classification methods and application of image processing on the ultrasound images of are aimed to categorize the US images for medical diagnosis purpose to make accept/reject decisions as per the international biomedical standards. The diagnosis scheme includes three steps: Preprocessing, feature extraction and classification. Finally experimental results are reported for three different classifiers (Support Vector Machine, ANFIS,CPNN).
\end{abstract}

\section{Keywords}

X Ray, Ultrasound Imaging, Preprocessing Techniques: Enhancement, Segmentation \& Classifiers CPNN, ANFIS \& SVM

\section{INTRODUCTION}

Ultrasound imaging is an important medical image to diagnose the patient, but it contains speckle noise which occur when the coherent and non-coherent detector are used to interrogate a medium which is rough on the scale of human visibility. The speckle is most often considered a dominant source of multiplicative in nature. The wide spread usage of ultrasound imaging equipment necessitates the need for better image processing technique to offer detailed image to the medical practitioner. However, sonography is much more operator-dependent, reading ultrasound image requires well-trained and experienced radiologists. Even well-trained experts may have a high inter-observer variation rate; therefore computer-aided improvement is needed to help radiologists in diseases detection and classification. There are many factors which can disturb the ultrasound image, such as: special speculative reflections, refractions, interferences, attenuation, distortions, non-linear propagation etc.

\section{RELATED WORK}

Hwanga et al. [1] , presented the diagnostic accuracy of focal liver lesions by evaluating key features of hemangiomas and cancerous lesions in ultrasound images. Focal liver lesions were divided into 29, 37 and 33 with severe hemangiomas. Total extract of hybrid features an ordered 42185 statistical measurement of resistance at 18 levels of gray and echogenicity. A total of 29 categories were chosen by a major breakdown of the components that are used as a set of inputs to the neural network to transmit energy. For the diagnosis of lesions were evaluated using forecast results positive, negative predictive value, sensitivity and accuracy. Results of the experiments showed that the proposed method proved highly effective, highly accurate more than $96 \%$ in the diagnosis of focal lesions (against hemoangioma, against malignant hemangiomas and anti-cancer) in the ultrasonic image. Accuracy slightly increased echogenicity include optimal sets of features.

To classify a given data set into a fixed number of clusters (assume $\mathrm{k}$ clusters), one for each cluster it defines $\mathrm{k}$ centroids. These centroids should be positioned sharply to obtain a global optimal solution. To place them as much as possible far away from each other is the best choice. Then coordinate each data point to any of these clusters having nearest centroid. Then re-calculate $\mathrm{k}$ new centroids as centers of the clusters and rebind the same data set points to nearest new centroid. Repeat this process either for a fixed number of repetitions or until two sub-sequent iteration having same centroids [2].

Mathematically, there are two basic models of noise, additive and multiplicative. Noise additive in nature is structured can be easily modelled, so they can be eliminated or reduced without much difficulty. While multiplicative noise is image independent, because of this they are complex to model and thus it is difficult to reduce. When the multiplicative noise seems to quality caused by dephasing of ecosystem disturbances, it is called "speckle noise" [3].

Qiangzhi Zhang et al [4] proposed to improve the mnogoobektivno robust graphic approach for dividing the part of the echo image of the breast. This approach is adopted three different target functions to achieve the purpose of the thirst. To combine efficiency and effectiveness, set the goal of three functional tricks taking a new benchmark among some crime sites. Then the algorithm for the optimization of the lens of the particle was made to optimize the performance of a robust method of segmental graphics. Theoretically, according to the definition of the variance between the classes, the variation in the average grade and slope, increasing their efficiency can overcome the problem of segmentation and paranoia, and to get the better of the outline of the breast tumor. 
K. Binaee and R.P.R. Hasanzadeh [5] proposed a new twisted filter that is divided into two parts, namely detection and filtration. In opening of the pixel is divided based on fuzzy rules the same side in the third, and completed and then filtered noise is applied to the art based on the similarity with the distance plane and the noise is filtered from the ultrasound image. Applying this method is minimized noise noise while maintaining detailed detail.

Okuwobi Idowu et.al [6] Exposure of the image using a practical wavelet technique. The author concludes that Wiener filters in denoising images exhausted all existing brightness levels that are used as a Sure Shrink, Visu Shrink, and the Bayes Shrink. The Wiener filter in the "Wavelet" domain works better than the Wiener Fourier filtering and filtering method. The adaptive thresholding is therefore better than the global thresholding. [6]

Haijuan $\mathrm{Hu}$ et al [7] worked on ejects of mixtures of noise and noise by means of plastic weight. First created, the rule of the large number and the theoretical point of distribution, to indicate the level of the point of the filter, this means to remove the gaussian noise. Based on the theoretical point for the point, this article proposes an average weighting plate of distortion filtering in its mixing with the Gaussian noise, and combining the basic concept of the triple filter and the filter for nonlinear means. Based on the theorem for this point, propose a new filter called Patch-based Filter (PWMF) that combats the mixed noise, which leads to the expansion of the NL Foundation.

Hossein Talebi [8] Bug fixes by a real world filter

development model, where each pixel is calculated from all pixels in the image. It is implemented in two steps. In the first step to obtain a statistical analysis of the proposed global filter based on the operator's spectral decomposition and study the impact of minimizing the spectral decomposition. Secondly, in order to obtain estimates to a Spectral component using the Nyström extension. Using these approaches, the author demonstrates that this universal filter can be achieved efficient fairly small fraction of the pixel in this figure [1]. Deepa Parasar proposed segmentation of foetus ultrasound image,(10).The research method drawback is that they have not used CPNN which is the fastest network also our accuracy determined is effective and efficiency is enhanced

1. The proposed algorithm flow chart is shown in fig

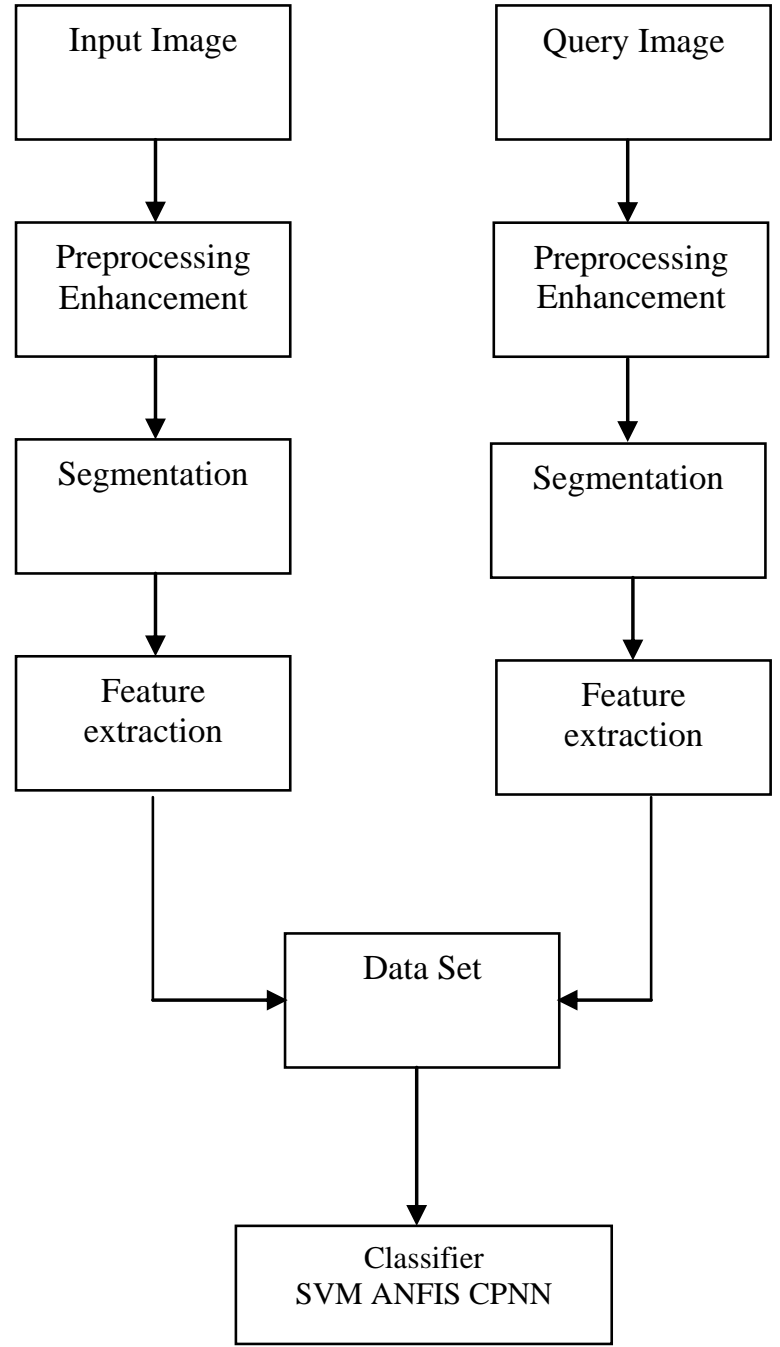

Fig1 Flow graph of computer aided decision support system

\section{COMPUTER AIDED DECISION SUPPORT SYSTEM}

In the computer aided automated recognition system, some image preprocessing techniques are used to remove noise and enhance the image quality of the image, here noise reduction is mandatory in the beginning and then contrast enhancement. Ioannis Valavanis[11] enhanced image first then gone for noise reduction, but it is not preferable since noise has to be reduced in the beginning before enhancement. Then concept of application of three segmentation techniques i.e. Particle swarm optimization based segmentation has been implemented on ultrasonic biomedicalimages for detection of abnormality, produced in liver, foetus,lungs. Geometric, related geometric and texture features are extracted and given as input to classifiers and identified accuracy [12-17].

\section{FEATURE EXTRACTION CLASSIFICATION 4.1 Texture Features}

The 14 most commonly used texture features are extracted from ultrasonic image can be defined and described. Texture feature used to partition images into regions of interest and to classify those regions, provides information in the spatial arrangement of color or intensities in an 
image, characterized by the spatial distribution of intensity levels in a neighborhood, repeating pattern of local variations in image intensity and cannot be defined for a point.

Texture is a repeating pattern of local variations in image intensity [12]

The purpose of feature extraction is to reduce the original data set by measuring certain properties, or features, that distinguish one input pattern from another pattern. The extracted feature should provide the characteristics of the input type to the classifier by considering the description of the relevant properties of the image into a feature space. 14 features based on the first order histogram and the gray level co-occurrence matrices (GLCM) have been used in this work. Co-occurrence matrix depicts Joint histogram of gray levels of a pair of pixels with a given spatial relationship. It captures the statistics of the gray level spatial variation. Co-occurrence matrices calculate the joint probability of adjacent pixels along a given direction $\theta$ having co-occurring values $i$ and j.[13]

Four matrices were calculated, for $\theta=0,45,90$, and 135 degrees, and combined in an averaged co-occurrence matrix since no directional variations in texture were expected. The 14 textural measures defined by Haralick were computed for each ultrasound image.

\section{ANGULAR SECOND MOMENT}

Angular Second Moment means energy or uniformity.It measures the number of repeated pixel pairs. The energy is expected high if the occurrence of repeated pixel pairs are high. The more homogeneous the image, the larger the value.

$p(i, j)=$ The normalized co-occurrence matrix

$\mathrm{Ng}=$ The number of discrete gray levels of the images.

$$
\sum_{i=1}^{N g} \sum_{j=1}^{N g}\{P(i, j)\}^{2}
$$

\section{CONTRAST}

It returns a measure of local intensity contrast of an image means how different the gray-level values in the pixel pair are. The contrast is expected low if the gray levels of each pixel pair are similar.

$$
\sum_{n=0}^{\forall g-1} n^{2}\left\{\sum_{i=j=n}^{n} i j\right\}
$$

3. CORRELATION

It measures the linear dependency of gray levels on those of neighboring pixels. Feature values range from -1 to 1 , these extremes indicating perfect negative and positive correlation respectively.

$$
\sum_{i=1}^{N g} \sum_{j=1}^{N g}\left(i-\mu_{X}\right)\left(j-\mu_{Y}\right) p(i, j) / \sigma_{X} \sigma_{Y}
$$

\section{VARIANCE}

It measures the dispersion around the mean of combinations of reference and neighbor pixels.

The Variance is expected large if the gray levels of the image are spread out.

$$
\sum_{i=1}^{N g} \sum_{j=1}^{N g}\left[(i-\mu)^{2} P(i, j)\right]
$$

\section{INVERSE DIFFERENCE MOMENT}

It provides the smoothness of the image.It is expected high if the gray levels of the pixel pairs are similar.

$$
\sum_{i=1}^{N g} \sum_{j=1}^{N g} 1 / 1+(i-j) p(i, j)
$$

\section{SUM AVERAGE}

It provides the mean of the gray levels in the image.

The Sum average is expected large if the sum of gray levels of the image is high.

$$
\sum_{i=2}^{2 N g}\left[i p_{x+y}(i)\right]
$$

7.SUM VARIANCE

$\sum_{i=2}^{2 N g}\left[(i-s u m a v e r a g e){ }^{2} p_{x+y}(i)\right]$

8.SUM ENTROPY

$$
-\sum_{i=2}^{N g}\left[\left(p_{x+y}(i) \operatorname{LOg} p_{x+y}(i)\right]\right.
$$

9. ENTROPY

It measures the level of spatial disorder of gray levels means the randomness of a gray level distribution.

The entropy is expected high if the gray levels are distributed randomly throughout the image.

$$
\sum_{i=1}^{N g} \sum_{j=1}^{N g}[(p(i, j) \log p(i, j)]
$$

\section{DIFFERENCE VARIANCE}

$$
P_{x y}=\sum_{i=0}^{N g=1}\left(i-f^{\prime}\right)^{2} p_{x-y(i)}
$$

11.DIFFERENCE ENTROPY

$$
-\sum_{i=0}^{N g-1}\left[\left(p_{x-y}(i) \log p_{x-y}(i)\right]\right.
$$

12. INFORMATION MEASURES OF CORRELATION

$$
\frac{\text { Hxy-Hxyl }}{\text { MAX[Hx, Hy] }}
$$

And $\left[1-\exp [-2 . \mathrm{O}(H x y 2-H x y)]^{\frac{1}{2}}\right.$

13. MAXIMAL CORRELATION COEFFICIENT

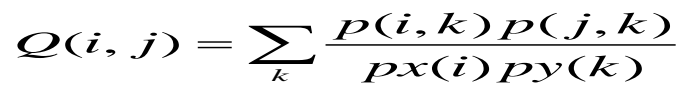

\section{HOMOGENEITY}

Measures the similarity of a pixel pair. If the gray levels of each pixel pair are similar the homogeneity is expected large just like inverse difference moment.

\section{SUM OF THE SQUARES:}




$$
\sum_{i=1}^{N g} \sum_{j=1}^{N g} p(i, j) / 1+(i-j)
$$

\section{CLASSIFIERS}

In this research work, counterpropagation neural network, support vector machine and artificial neuro fuzzy inference system are analyzed for the classification applications.

\subsection{Conventional CPN}

The CPN neural network is a hybrid neural network which employs both the supervised and unsupervised training methodologies. It consists of the input Kohonen layer which uses the "winner take-all" strategy and the output Grossberg layer which uses the error signal for weight adjustment. The error signal is used to update only the output layer weights unlike back propagation network where error is used to update weights of both the layer. Thus, this network is named as Counter Propagation Neural Network to show that it is contrary to the conventional BPN.[9].

A CPN consists of three layers: the input layer, the competition layer and the output layer.

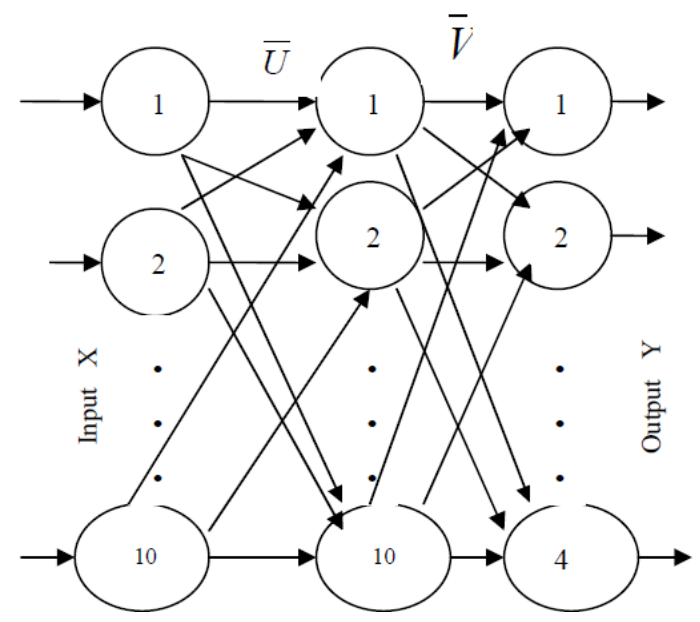

Fig2 CPN layers

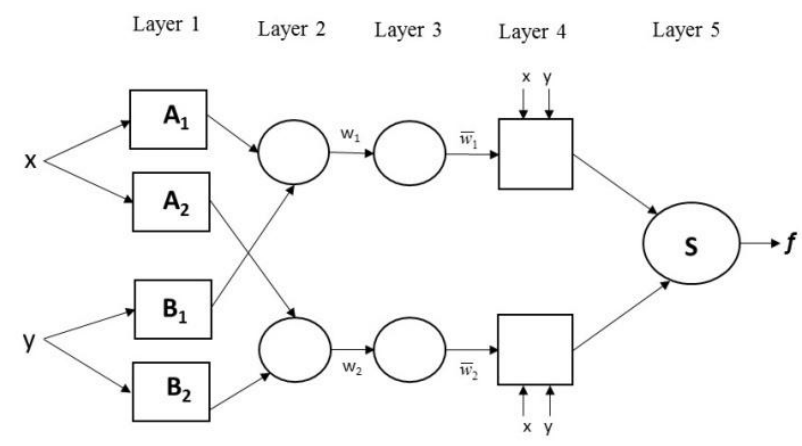

Fig 3: ANFIS Architecture

\subsection{Adaptive Neuro-Fuzzy Inference System (ANFIS) Classification}

ANFIS is part of theneuro-fuzzy inference hybrid system and the intricate work of the Sugenotype fuzzy inference system, which was developed by the Jang [200]. ANFIS has a similar Multilayer feed forward neural network structure for feeding information, but in ANFIS the link only shows the direction of the flow of signals between nodes and does not tie any weights to the links.

\subsubsection{ANFIS Structure}

ANFIS architecture consists of five layers of nodes. Out of the five layers, the first and the fourth layers consist of adaptive nodes while the second, third and fifth layers consist of fixed nodes. The adaptive nodes are associated with their respective parameters, get duly updated with each subsequent iterations while the fixed nodes are devoid of any parameters shown in fig $3[11,12]$.

Rule 1: $\mathrm{f} 1=\mathrm{p} 1 \mathrm{x}+\mathrm{q} 1 \mathrm{y}+\mathrm{r} 1$ if $(\mathrm{x}$ is $\mathrm{A} 1)$ and (y is B1)

Rule 2: $f 2=p 2 x+q 2 y+r 2$ if ( $x$ is $A 2)$ and (y is B2)

where fi are the outputs within the fuzzy region specified by the fuzzy rule, $\mathrm{x}$ and $\mathrm{y}$ are the inputs, $\mathrm{Ai}$ and $\mathrm{Bi}$ are the fuzzy sets, , pi, qi and ri are the design constraints that are determinedduring the training process. Implementation of these two rules for the ANFIS architecture is shown in Figure 5.3, in which a circle specifies a fixed node, whereas a square specifies an adaptive node.

\subsection{Support Vector Machine Classification}

Support Vector Machine (SVM) is a discriminative classifier formally defined by a separating hyperplane. The aim of SVM is to find a decision plane that has a maximum distance (margin) from the nearest training pattern [12]. In order to get the required decision plane, SVM maps the feature vector to a higher-dimensional space. In this space a hyperplane is found by SVM to separate the two classes with a decision boundary set by support vectors [12]. The high computational cost of mapping process can be reduced by an appropriate kernel. In this paper, a binary SVM classifier is adopted using the polynomial kernel. The SVM can be put in the form of a constrained optimization problem given a total of $\mathrm{N}$ training vectors denoted by $\mathrm{xi}$ with a corresponding class

$$
\max Q(\lambda)=\sum_{i=1}^{N} \lambda_{i}-1 / 2 \sum_{i=1}^{N} \sum_{j=1}^{N} \lambda_{j} y_{i} y_{j} K\left(x_{i}, x_{j}\right)
$$

Subject to $\sum_{i}^{N} \lambda_{i} y_{i}=0 \lambda_{i} \geq 0$

In this optimization problem, $\lambda_{i} \square$ is the Lagrange multiplier.

$\mathrm{K}\left(\mathrm{x}_{\mathrm{i}}, \mathrm{x}_{\mathrm{j}}\right)$ illustrates the kernel function and can be written $\operatorname{asK}\left(\mathrm{x}_{\mathrm{i}}, \mathrm{x}_{\mathrm{j}}\right)=\Phi\left(\mathrm{x}_{\mathrm{i}}\right)^{\mathrm{T}} \Phi\left(\mathrm{x}_{\mathrm{j}}\right)$, with $\Phi\left(\mathrm{x}_{\mathrm{i}}\right)$ being the function which transforms $\mathrm{x}_{\mathrm{i}}$ to a high dimensional space.

\section{RESULTS AND DISCUSSIONS}

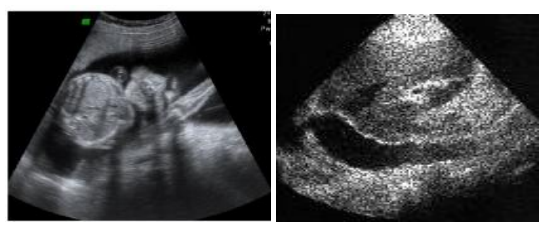

Input Image a \& b 

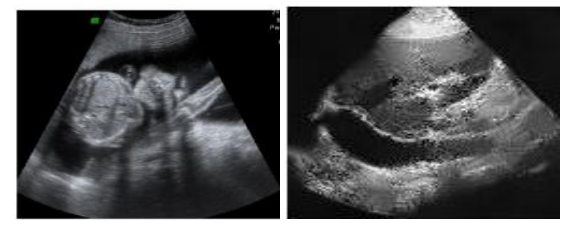

Noise free Image $\mathrm{c} \& \mathrm{~d}$

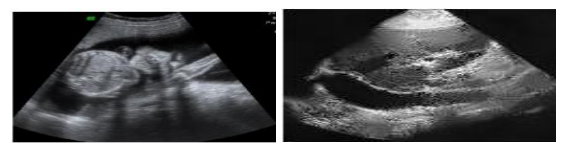

Segmented Image e \& f

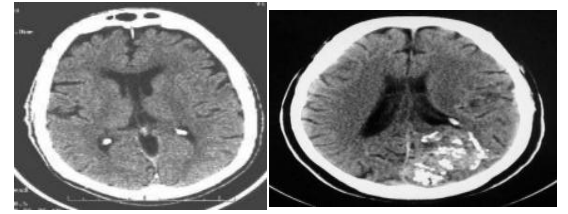

Segmented Image $\mathrm{g} \& \mathrm{~h}$

Table I: Proposed Classification method Performance

Measures

\begin{tabular}{|l|l|l|l|l|}
\hline & $\begin{array}{l}\text { Accuracy } \\
(\%)\end{array}$ & $\begin{array}{l}\text { Sensitivity } \\
(\%)\end{array}$ & $\begin{array}{l}\text { PPV } \\
(\%)\end{array}$ & $\begin{array}{l}\text { Specificity } \\
(\%)\end{array}$ \\
\hline ANFIS & 89 & 81.4 & 92.4 & 91.4 \\
\hline SVM & 90 & 82 & 94.8 & 93.5 \\
\hline CPNN & 92 & 93.23 & 95.1 & 95.65 \\
\hline
\end{tabular}

\section{CONCLUSION AND FUTURE SCOPE}

The main focus of proposed system is enhancement of biomedical image for analysis purpose by fuzzy filter integrated with Particle swarm optimization K-mean Clustering segmentation..The method which is proposed is innovative compared to the state of art, counter propagation neural network (CPNN) has been never tried but our research paper innovated $92 \%$ accuracy compared to other classifiers.Future work can be extended to do classification of ultrasound image \& comparative analysis with various types of classifiers with variations in the features.

\section{REFERENCES}

[1] Hwanga Y N, Lee JH, Kim GY, Jiang YY and Kim SM "Classification of focal liver lesions on ultrasound images by extracting hybrid textural features and using an artificial neural network", Bio-Medical Materials and Engineering, Shanghai-China , pp. S1599-S1611, 2015

[2] E.Setiawati, Adiwijaya and Tjokorda A.B.W. "Particle Swarm Optimization on Follicles Segmentation to Support PCOS Detection" 2015 3rd International Conference on Information and Communication Technology (ICoICT).pp.371-376,2015.

[3] Qiangzhi Zhang, Xia Zhao, Qinghua Huang, "A MultiObjectively-Optimized Graph-Based Segmentation Method for Breast Ultrasound Image", IEEE, pp. 116120,2014

[4] Qiangzhi Zhang, Xia Zhao, Qinghua Huang” A MultiObjectively-Optimized Graph-Based Segmentation
Method for Breast Ultrasound Image”, IEEE, pp. 116120,2014 .

[5] K. Binaee, R.P.R. Hasanzadeh, "An ultrasound image method using local gradient based fuzzy similarity", Biomedical enhancement Signal Processing and control 13, Elsevier, pp. 89-101,2014.

[6] Okuwobi Idowu, Paul Yonghua Lu, "Image Denoising Using Wavelet Thresholding Techniques", International Journal of Education and Research Vol. No. 2 February 2014page no. 1-5.

[7] Haijuan $\mathrm{Hu}$, Bing Li, Quansheng Liu , "Removing Mixture of Gaussian and Impulse Noise by PatchBased Weighted Means", Preprint submitted to Elsevier arXiv:1403.2482v1 [cs.CV] 11 Mar 2014 page no. 1-29.

[8] Hossein Talebi, Student Member, IEEE, and Peyman Milanfar, Fellow, IEEE: "Global Image Denoising" IEEE Transactions On Image Processing, VOL. 23, NO. 2, FEBRUARY 2014 page no. 755-768.

[9] A.P. Dhawan, Medical Image Analysis.: John Wiley Publication and IEEE Press, 2003.

[10] Deepa Parasar "Ultrasound Image Enhancement using ImprovedFixed Point Independent Component Analysis," International Conference on Electrical, Electronics, Signals, Communication and Optimization (EESCO) - 2015.

[11] IoannisValavanis,et al,"Multiclass defect detection and classification in weld radiographic images using geometric and texture features" Expert Systems with Applications Volume 37, Issue 12, Pages 7606-7614 July, 2010

[12] H. Kasban, et.al," Welding defect detection from radiography images with a cepstral approach", NDT and E International, doi: 10.1016 (In the press October 2010).

[13] N.M.Nanditha, et al, "Comparative study on the suitability of feature extraction techniques for tungusten inclusion and hotspot detection from weld thermographs for online weld montoring." International Journal of Engineering and Material science, Vol. 17 pp. 20-26, February 2010.

[14] S. Margret Anouncia, et al "A knowledge model for gray image interpretation with emphasis on welding defect classification," Computer Industry, vol. 61, pp. 742-749, October 2010.

[15] P.N.Jebarani et al, "Automatic detection of weld defects in pressure vessels using fuzzy neural network," International Journal of Computer Applications, vol. 1, no. 39, pp. 0975-8887, 2010.

[16] P.N.Jebarani et al, "Automatic detection of porosity and slag inclusion in boiler using statistical pattern recognition technique ," International Journal of Computer Applications, vol. 1, no. 39, pp. 0975-8887, 2010.

[17] Rafael Vilar, et al, "An automatic system of classification of weld defects in radiographic images", NDT \& E International Volume 42, Issue 5, Pages 467-476 July 2009. 